\title{
Assessment of glutamatergic synaptic transmission and plasticity in brain slices: relevance to bioelectronic approaches
}

\author{
Eric H. Chang ${ }^{1,2,4}$, Samantha T. Carreiro ${ }^{3}$, Stephen A. Frattini ${ }^{1}$ and Patricio T. Huerta ${ }^{1,4^{*}}$ (D)
}

\begin{abstract}
Background: Glutamatergic neurons represent the largest neuronal class in the brain and are responsible for the bulk of excitatory synaptic transmission and plasticity. Abnormalities in glutamatergic neurons are linked to several brain disorders and their modulation represents a potential opportunity for emerging bioelectronic medicine (BEM) approaches. Here, we have used a set of electrophysiological assays to identify the effect of the pyrimidine nucleoside uridine on glutamatergic systems in ex vivo brain slices. An improved understanding of glutamatergic synaptic transmission and plasticity, through this type of examination, is critical to the development of potential neuromodulation strategies.
\end{abstract}

Methods: Ex vivo hippocampal slices ( $400 \mu \mathrm{m}$ thick) were prepared from mouse brain. We recorded field excitatory postsynaptic potentials (fEPSP) in the CA1's stratum radiatum by stimulation of the CA3 Schaeffer collateral/ commissural axons. Uridine was applied at concentrations (3, 30, 300 $\mu \mathrm{M})$ representing the physiological range present in brain tissue. Synaptic function was studied with input-output (I-O) functions, as well as paired-pulse facilitation (PPF). Synaptic plasticity was studied by applying tetanic stimulation to induce post-tetanic potentiation (PTP), short-term potentiation (STP) and long-term potentiation (LTP). Additionally, we determined whether uridine affected synaptic responses carried solely by $n$-methyl-d-aspartate receptors (NMDARs), particularly during the oxygenglucose deprivation (OGD) paradigm.

Results: The presence of uridine altered glutamatergic synaptic transmission and plasticity. We found that uridine affected STP and LTP in a concentration-dependent manner. Low-dose uridine ( $3 \mu \mathrm{M})$ had no effect, but higher doses (30 and $300 \mu \mathrm{M}$ ) impaired STP and LTP. Moreover, uridine $(300 \mu \mathrm{M})$ decreased NMDAR-mediated synaptic responses. Conversely, uridine (at all concentrations tested) had a negligible effect on PPF and basal synaptic transmission, which is mediated primarily by a-amino-3-hydroxy-5-methyl-4-isoxazolepropionic acid receptors (AMPARs). In addition, uridine $(100 \mu \mathrm{M})$ exerted a protective effect when the hippocampal slices were challenged with OGD, a widely used model of cerebral ischemia.

\footnotetext{
* Correspondence: pato.huerta@gmail.com

${ }^{1}$ Laboratory of Immune \& Neural Networks, Institutes of Molecular Medicine

and Bioelectronic Medicine, Feinstein Institutes for Medical Research,

Northwell Health, 350 Community Drive, Manhasset, NY 11030, USA

${ }^{4}$ Department of Molecular Medicine, Zucker School of Medicine at Hofstra/

Northwell, 500 Hofstra Blvd, Hempstead, NY 11549, USA

Full list of author information is available at the end of the article
}

(c) The Author(s). 2019 Open Access This article is distributed under the terms of the Creative Commons Attribution 4.0 International License (http://creativecommons.org/licenses/by/4.0/), which permits unrestricted use, distribution, and reproduction in any medium, provided you give appropriate credit to the original author(s) and the source, provide a link to the Creative Commons license, and indicate if changes were made. The Creative Commons Public Domain Dedication waiver (http://creativecommons.org/publicdomain/zero/1.0/) applies to the data made available in this article, unless otherwise stated. 
(Continued from previous page)

Conclusions: Using a wide set of electrophysiological assays, we identify that uridine interacts with glutamatergic neurons to alter NMDAR-mediated responses, impair synaptic STP and LTP in a dose-dependent manner, and has a protective effect against OGD insult. This work outlines a strategy to identify deficits in glutamatergic mechanisms for signaling and plasticity that may be critical for targeting these same systems with BEM device-based approaches. To improve the efficacy of potential neuromodulation approaches for treating brain dysfunction, we need to improve our understanding of glutamatergic systems in the brain, including the effects of modulators such as uridine.

Keywords: Uridine, Nucleoside, LTP, Synaptic plasticity, Glutamate, NMDA

\section{Background}

Bioelectronic medicine encompasses a set of technologies that harness the electrical nerve impulses of the body to treat disease. The current approaches have mainly focused on electrical stimulation of the peripheral nervous system, but there is also potential of employing the principles of synaptic function, synaptic plasticity, and brain biochemistry for the implementation of bioelectronic approaches in the CNS. Glutamate is the principal excitatory neurotransmitter in the brain. It is released from the presynaptic terminals of pyramidal neurons and it binds to glutamate receptors that are located in the postsynaptic neurons. There are three classes of ionotropic glutamate receptors, namely NMDARs, AMPARs and kainate receptors, which have a role not just in excitatory synaptic transmission but also in synaptic plasticity and higher cognitive functions. Importantly, abnormal elevations of glutamate can induce neurotoxicity, and because of this, glutamate has been implicated as a potential contributor to the pathogenesis of several neurodegenerative disorders. In this study, we aimed to investigate whether uridine is capable of altering glutamatergic synaptic transmission and synaptic plasticity with the use of ex vivo hippocampal slices and electrophysiological recordings. The hippocampal slice is an ideal preparation because it maintains many of the functions that neurons perform in vivo and it preserves the local synaptic circuitry. Therefore, brain slices are a good system in which to evaluate the molecular changes associated with drug treatment or by external neuromodulation, such as via direct current stimulation (e.g., transcranial direct current stimulation or deep brain stimulation). Moreover, hippocampal slices are able to sustain glutamatergic synaptic plasticity, which is usually tested with the paradigm of LTP. Extensive research has shown that LTP represents a form of synaptic plasticity that is inputspecific, associative, and widely accepted as a synaptic model of memory formation (Bliss and Lomo, 1973; Bliss and Collingridge, 1993). In addition, it has been shown that brain slices subjected to a brief OGD injury exhibit regionally selective death of pyramidal neurons in the CA1 region, and have been used to model different brain disorders (Cho et al., 2007).

To test whether glutamatergic signaling and plasticity can be affected by non-traditional neuromodulators, we applied the nucleoside uridine on ex vivo brain slices during a broad set of electrophysiological measurements. Uridine is a building block of ribonucleic acid (RNA), which makes it an essential molecule for cell metabolism. Several decades of research have shown that uridine might have other functions in brain cells, besides being a component of nucleic acids. For instance, uridine is the only source of cytidine, which is a building block of phosphatidylcholine, one of the key phospholipids within the cell membrane (Dawson 1968; Wang et al., 2007). Some studies have shown that uridine added to neuronal cultures is capable of stimulating dendritic branching, thus increasing the number of dendrites per cell. This effect is thought to result from enhancing phosphatidylcholine synthesis, which adds new cell membrane, but also from blocking the receptors that stop dendrites from growing (Pooler et al., 2005; Silei et al., 2000). Notably, it has been shown that orally administered uridine5 -monophosphate given to aged rats supports an increased release of dopamine in the striatum (35\% over control level) and dendritic outgrowth, demonstrating that, even in old animals, oral uridine intake can support neurotransmitter release and dendritic branching in vivo (Wang et al., 2005). While little is known about the effects of uridine on neurophysiology, a few studies have shown that it can work as an anticonvulsant in animal models of epilepsy (Slezia et al., 2004; Zhao et al., 2006, 2008). In regards to neurotransmitter interactions, uridine has been reported to bind competitively to gamma-aminobutyric acid (GABA) receptors (Guarneri et al., 1985) and to be released following seizures (Slezia et al., 2004), suggesting a generally inhibitory effect on synapses.

Uridine supplementation has been investigated in a number of animal models for brain disease, including epilepsy (Zhao et al., 2006; Zhao et al., 2008), Huntington's disease (Saydoff et al., 2006), traumatic brain injury 
(Kabadi and Maher, 2010) Parkinson's disease (Cansev et al., 2008), cognitive deficit (De Bruin et al., 2003), amyotrophic lateral sclerosis (Amante et al., 2010), and depression-like syndromes (Carlezon et al., 2002, 2005). Together, these results suggest that uridine is an attractive therapeutic candidate in the treatment of several brain illnesses and has an effect on brain function (Wurtman et al., 2010), although the neurophysiological basis of this effect remains to be elucidated.

Circulating plasma levels of uridine in humans range from 3 to $8 \mu \mathrm{M}$, but can reach concentrations of $150 \mu \mathrm{M}$ under multiple dosing regimens (van Groeningen et al., 1991; Weinberg et al., 2010). Basal plasma uridine levels in rodents are comparable to those in humans, but within the brain, concentrations can reach the 100$300 \mu \mathrm{M}$ range, with maximal concentrations $>350 \mu \mathrm{M}$ after intraperitoneal dosing (Amante et al., 2010). Based on these prior findings, we decided to test three different concentrations $(3 \mu \mathrm{M}, 30 \mu \mathrm{M}, 300 \mu \mathrm{M})$ of uridine for their ability to alter glutamatergic transmission and plasticity. We find that basal synaptic transmission is unaltered by the three tested concentrations, but long-term synaptic plasticity is impaired at the two higher concentrations $(30 \mu \mathrm{M}$ and $300 \mu \mathrm{M})$. Through the pharmacological isolation of NMDAR-mediated responses, we identify that uridine has specific effects on NMDARs in the hippocampus. We also find that uridine $(100 \mu \mathrm{M})$ has a protective effect in an ex vivo model of ischemia.

\section{Methods}

\section{Experimental animals}

All animals used in this study were female BALB/cJ mice (The Jackson Laboratory, Bar Harbor, ME) of 3-8 months of age. Mice had ad libitum access to food and water, and were maintained in strict accordance with the recommendations in the Guide for the Care and Use of Laboratory Animals of the National Institutes of Health. The local Institutional Animal Care and Use Committee (Feinstein Institute for Medical Research) approved the animal protocol. All efforts were made to minimize and ameliorate suffering and pain to animals used in this study.

\section{Ex vivo hippocampal slice preparation}

$\mathrm{BALB} / \mathrm{cJ}$ mice were anaesthetized with isoflurane in a closed container, then immediately decapitated. The brain was quickly extracted into ice-cold $\left(<2{ }^{\circ} \mathrm{C}\right)$ artificial cerebral spinal fluid (ACSF) that contained (in $\mathrm{mM}$ ): $126 \mathrm{NaCl}, 26 \mathrm{NaHCO}_{3}, 10$ glucose, $2.5 \mathrm{KCl}, 2.4 \mathrm{CaCl}_{2}$, $1.3 \mathrm{MgCl}_{2}, 1.2 \mathrm{NaH}_{2} \mathrm{PO}_{4}$ and was continuously gassed with $95 \% \mathrm{O}_{2}, 5 \% \mathrm{CO}_{2}$. Kynurenic acid $(1 \mathrm{mM})$, which is a non-specific blocker of excitatory amino acid receptors, was added to the ACSF during the dissection and slicing procedures. The brain was then bisected and both hemispheres were mounted onto a block with ethyl cyanoacrylate glue. Transverse hippocampal slices $(400 \mu \mathrm{m}$ thick) were prepared using a Leica VT1200 brain slicer. Brain slices were incubated for $35 \mathrm{~min}$ in ACSF at $35^{\circ} \mathrm{C}$, followed by $120 \mathrm{~min}$ in ACSF at $24^{\circ} \mathrm{C}$. Each slice was transferred to a recording chamber, continuously perfused with ACSF at $30^{\circ} \mathrm{C}$, for electrophysiological studies.

\section{Hippocampal electrophysiology}

Field excitatory postsynaptic potentials (fEPSP) were recorded with borosilicate glass electrodes $(2-3 \mathrm{M} \Omega$ tip resistance) placed in CA1's stratum radiatum at the midpoint between two bipolar stimulating electrodes (Frederick Haer \& Co, Bowdoinham, ME) that were placed to activate the Schaeffer collateral/commissural axons. This setup allowed for the recording of two independent pathways (test and control) in the same slice. The initial slope of the fEPSP was used as a measure of the postsynaptic response. fEPSP responses were amplified (AM Systems 1800), digitized at $10 \mathrm{kHz}$, and stored on a PC running custom software (written with AxoBasic, Axon Instruments, Union City, CA). For obtaining I$\mathrm{O}$ functions, the stimulation was reduced to a value at which no fEPSP was evoked. The stimulation was then increased incrementally to evoke steeper and larger fEPSPs. This was done until the appearance of a population spike, which reflected action potentials, generated by CA1 pyramidal cells, and defined the final point of the I-O function. The protocol for PPF involved activating the afferent axons with two stimulating pulses within a short $(<1 \mathrm{~s})$ inter-pulse interval (IPI). The IPIs were (in msec): 20, 50, 100, 200, 300, and 400. The pairedpulse ratio was calculated as the slope of the second fEPSP (P2) divided by the slope of the first fEPSP (P1). For plasticity experiments, a stable baseline was obtained for at least $15 \mathrm{~min}$. The baseline intensity was set to obtain a fEPSP slope that was half-maximal, as determined by I-O functions. Synaptic plasticity was induced by high-frequency stimulation (HFS), which consisted of either a tetanus train $(100 \mathrm{~Hz}$ for $1 \mathrm{~s})$ or theta burst stimulation (TBS, 10 trains of 4 pulses at $100 \mathrm{~Hz}$, with 200 msec between trains). We calculated three plasticity time-points, identified as PTP (measured from 6 responses at $1 \mathrm{~min}$ post-HFS), STP (measured from 30 responses at 10-15 min post-HFS) and LTP (measured from 30 responses at $40-45$ min post-HFS). For all LTP experiments, picrotoxin $(100 \mu \mathrm{M})$ was added to block $\mathrm{GABA}_{\mathrm{A}}$ receptors. A Good Laboratory Practice (GLP) lot of ultrapure uridine ( $\mathrm{MW}=244.2)$ was provided by Repligen Corporation (Waltham, MA). In order to analyze the temporal summation that occurred during the TBS, we used Origin (OriginLab, Northampton, MA) software to integrate the total depolarization area 
of each fEPSP response during the first TBS stimulation event.

For recording NMDAR-mediated fEPSPs, we used a magnesium-free ACSF solution containing 6-cyano-7nitroquinoxaline-2,3-dione (CNQX, $10 \mu \mathrm{M})$, and glycine $(30 \mu \mathrm{M})$. Baseline NMDAR-mediated fEPSPs were acquired and analyzed once every $20 \mathrm{~s}$ using WinLTP 2.01 software (WinLTP, Bristol, UK). For the OGD experiments, the brain slices were introduced into the recording chamber with ACSF + uridine $(100 \mu \mathrm{M})$ for the indicated incubation period. At the end of the incubation period, the ACSF solution was switched to an OGD solution that was identical to the normal ACSF except that it did not contain glucose and was bubbled with $100 \% \mathrm{~N}_{2}$ instead of $95 \% \mathrm{O}_{2}, 5 \% \mathrm{CO}_{2}$. This OGD solution perfused the chamber for a period of $6 \mathrm{~min}$, followed by normal ACSF for the remainder of the experiment.

\section{Statistical analysis}

Data are presented as mean \pm SEM, as indicated. To examine statistical significance, which was defined as $P<0.05$, we used factorial ANOVA, repeated measures ANOVA, and Student's t-test in samples that were normally distributed. We also used nonparametric tests, namely MannWhitney U (MWU) test and Kolmogorov-Smirnov test, in samples that were not normally distributed.

\section{Results}

Null effect of uridine on basal synaptic transmission

I-O functions indicated that uridine did not have an effect on basal synaptic transmission at any of the concentrations tested (Fig. 1a, b). The range of uridine concentrations $(3 \mu \mathrm{M}, 30 \mu \mathrm{M}, 300 \mu \mathrm{M})$ was chosen to represent the wide physiological range that brain tissue is exposed to in vivo, based on previous work (Amante et al., 2010). The I-O functions were compared using ANOVA with fiber volley amplitude as the repeated measure. This test showed that fEPSP slopes were similar across the range of concentrations tested: $3 \mu \mathrm{M}, \quad F_{9,129}=0.47, \quad P=0.51$; $30 \mu \mathrm{M}, F_{9,162}=0.46, P=0.51 ; 300 \mu \mathrm{M}, F_{9,162}=1.53, P=$ 0.24 . Uridine also had no significant effect on the slope of baseline fEPSPs when introduced into the recording
A

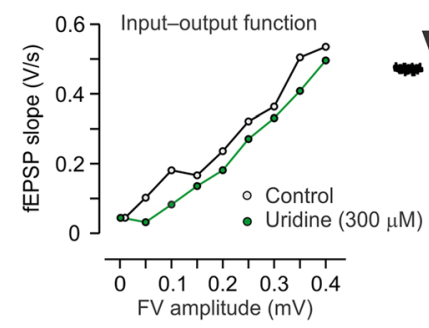

B

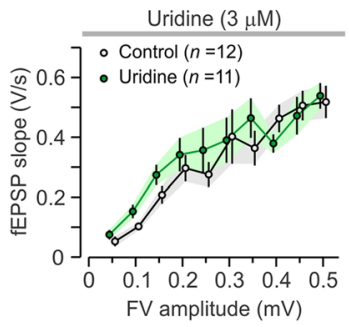

C

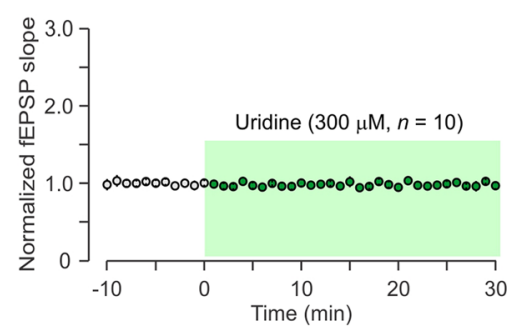

Control
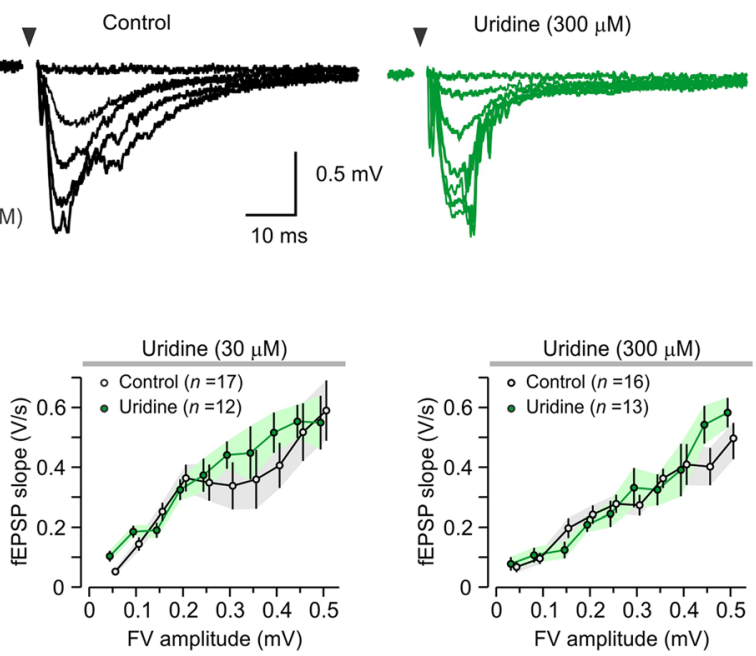

D

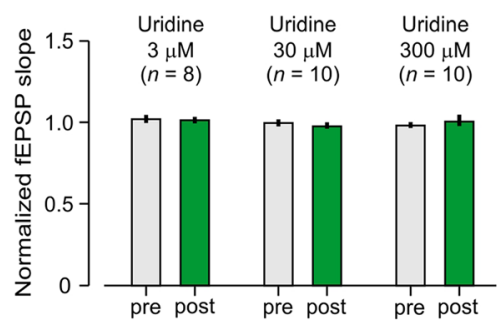

Fig. 1 Null effect of uridine on basal synaptic transmission. a Left, representative input-output (I-O) experiments for uridine (300 $\mu M$ ) and control; with the amplitude of the fiber volley (FV) as the independent variable and the slope of the fEPSP as the dependent variable. Right, sample overlaid traces from single I-O experiments. Electrical stimulation artifacts have been removed and are marked by arrowheads. $\mathbf{b}$ Plots of I-O responses (mean \pm SEM) indicate that basal synaptic transmission is not affected by any of the uridine concentrations tested. c Representative experiment showing that the fEPSP slope remains unchanged when uridine $(300 \mu \mathrm{M})$ is added to the brain slice placed in the recording chamber. $\mathbf{d}$ Normalized fEPSP slope (mean \pm SEM) showing that uridine $(3 \mu \mathrm{M}, 30 \mu \mathrm{M}$, and $300 \mu \mathrm{M})$ does not cause changes in field synaptic potentials, when measured 30 min post-application 
solution (Fig. 1c, d). These results indicate that uridine did not affect the strength of basal synaptic transmission across the population of hippocampal synapses.

\section{Null effect of uridine on PPF}

We tested short-term synaptic plasticity with the PPF protocol (Fig. 2a). This paradigm is designed to identify changes in the population of presynaptic terminals by using a pair of stimulating pulses within a short interpulse interval (Zucker 1989). PPF profiles were compared using ANOVA with inter-pulse interval as the repeated measure (Fig. 2b). This analysis showed that

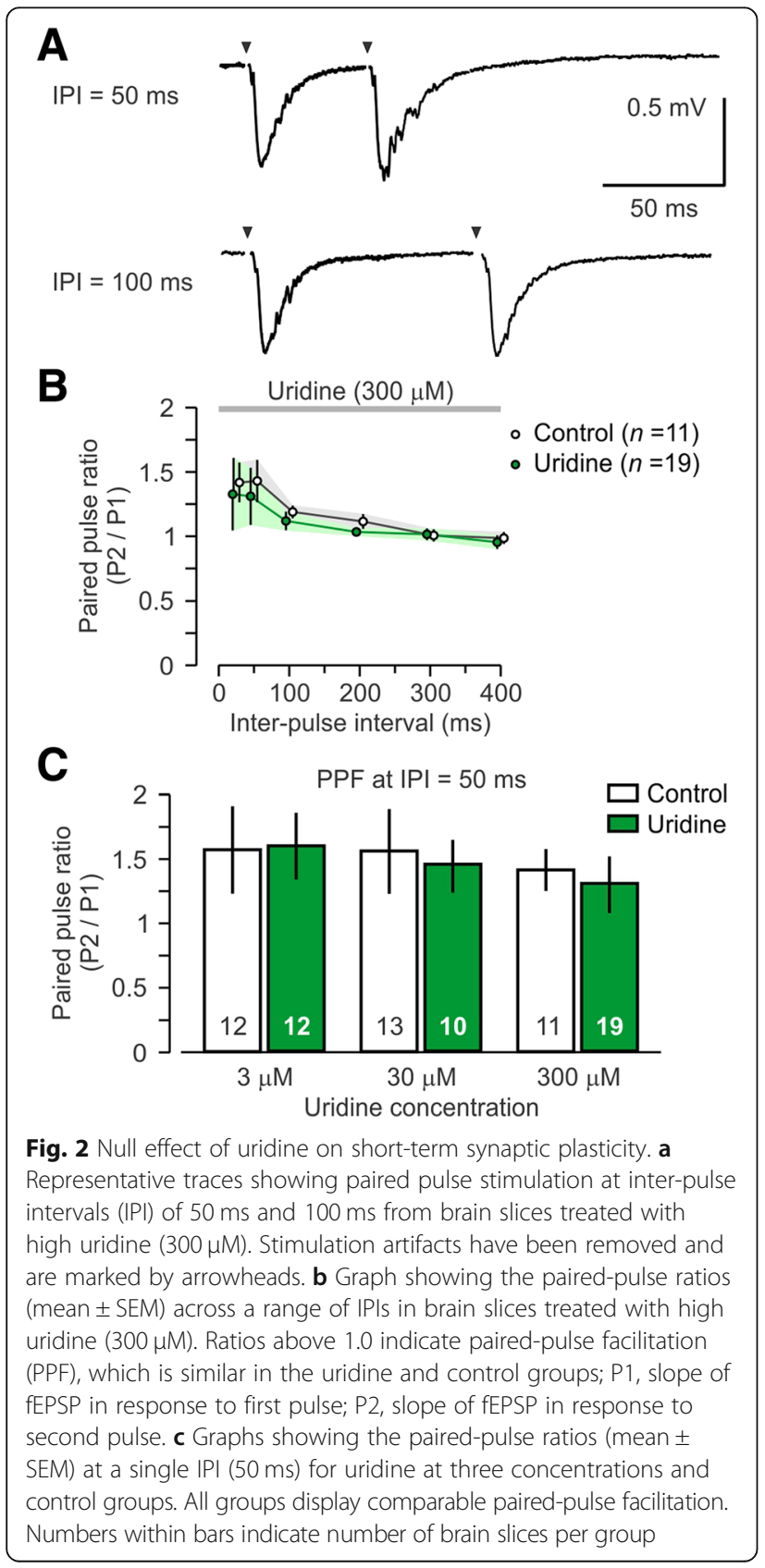

there were no differences in PPF across the range of concentrations tested (Fig. $2 \mathrm{c}$ ): $3 \mu \mathrm{M}, F_{5}, 115=0.65, P=$ $0.80 ; 30 \mu \mathrm{M}, F_{5,56}=3.09, P=0.13 ; 300 \mu \mathrm{M}, F_{5}, 85=0.39$, $P=0.55$. This indicated that short-term synaptic plasticity was unaffected by uridine.

\section{Effect of uridine on STP and LTP}

Synaptic plasticity was assessed by recording fEPSPs for a baseline period (15 min) and then applying HFS, which is well-known trigger for LTP. Brain slices exposed to low uridine $(3 \mu \mathrm{M})$ did not show significant differences in their LTP level from control brain slices (Fig. 3a, control, $155 \% \pm 3 \%$; uridine, $140 \% \pm 2 \%$ of baseline values; $T=1.60, P=0.11, \mathrm{t}$-test). There were also no differences in other plasticity time-points such as PTP (control, 198\% $\pm 5 \%$; uridine, $216 \% \pm 12 \% ; \quad T=1.96$, $P=0.07, t$-test) and STP (control, $175 \% \pm 9 \%$; uridine, $160 \% \pm 4 \% ; T=1.34, P=0.18$, t-test).

Brain slices exposed to the middle level of uridine $(30 \mu \mathrm{M})$ exhibited a significant difference in LTP (Fig. $3 \mathrm{~b}$, control, $144 \% \pm 6 \%$; uridine, $106 \% \pm 3 \%$; $T=4.30$, $P<0.0001$, t-test) and STP (control, $167 \% \pm 6 \%$; uridine, $134 \% \pm 4 \% ; T=4.34, P<0.0005$, t-test $)$, but no difference in PTP (control, 197\% $\pm 9 \%$; uridine, $174 \% \pm 6 \%$; $T=$ $0.98, P=0.33$, t-test). Brain slices exposed to high uridine $(300 \mu \mathrm{M})$ showed the most dramatic impairment in synaptic plasticity with differences in LTP (Fig. 3c, control, $147 \% \pm 2 \%$; uridine, $97 \% \pm 1 \% ; T=6.55, P<0.0001$, t-test), STP (control, $194 \% \pm 2 \%$; uridine, $112 \% \pm 1 \%$; $T=5.79$, $P<0.0001$, t-test), and PTP (control, $246 \% \pm 18 \%$; uridine, $142 \% \pm 7 \% ; T=5.25, P<0.0001$, t-test).

We next addressed the question of whether uridine affected the expression of LTP. We tested this by introducing uridine, starting at $10 \mathrm{~min}$ post-HFS, and measuring whether a 35-min period of drug application altered the level of potentiation (Fig. 3d). We found that uridine applied following the HFS did not have any effect on LTP expression at any of the concentrations we tested (Fig. 3e). Statistical comparison against control brain slices revealed no significant differences among groups $(3 \mu \mathrm{M}$, $T=0.50, P=0.62 ; 30 \mu \mathrm{M}, T=1.81, P=0.09 ; 300 \mu \mathrm{M}, T=$ $1.86, P=0.07$, t-tests).

\section{Burst analysis reveals lower total depolarization in the presence of uridine}

NMDAR-mediated synaptic responses have a long duration $(>100 \mathrm{~ms})$ so that they summate effectively under high frequency stimulation paradigms (higher than $10 \mathrm{~Hz}$ ) . By measuring the total depolarization value during the LTP-inducing tetanic stimulation, we obtained an indirect measure of this NMDAR-mediated response. Analysis of responses during the first TBS event of each tetanus (Fig. 4a) indicated that the mean total depolarization was not different at the low concentration of uridine $(3 \mu \mathrm{M}$; 

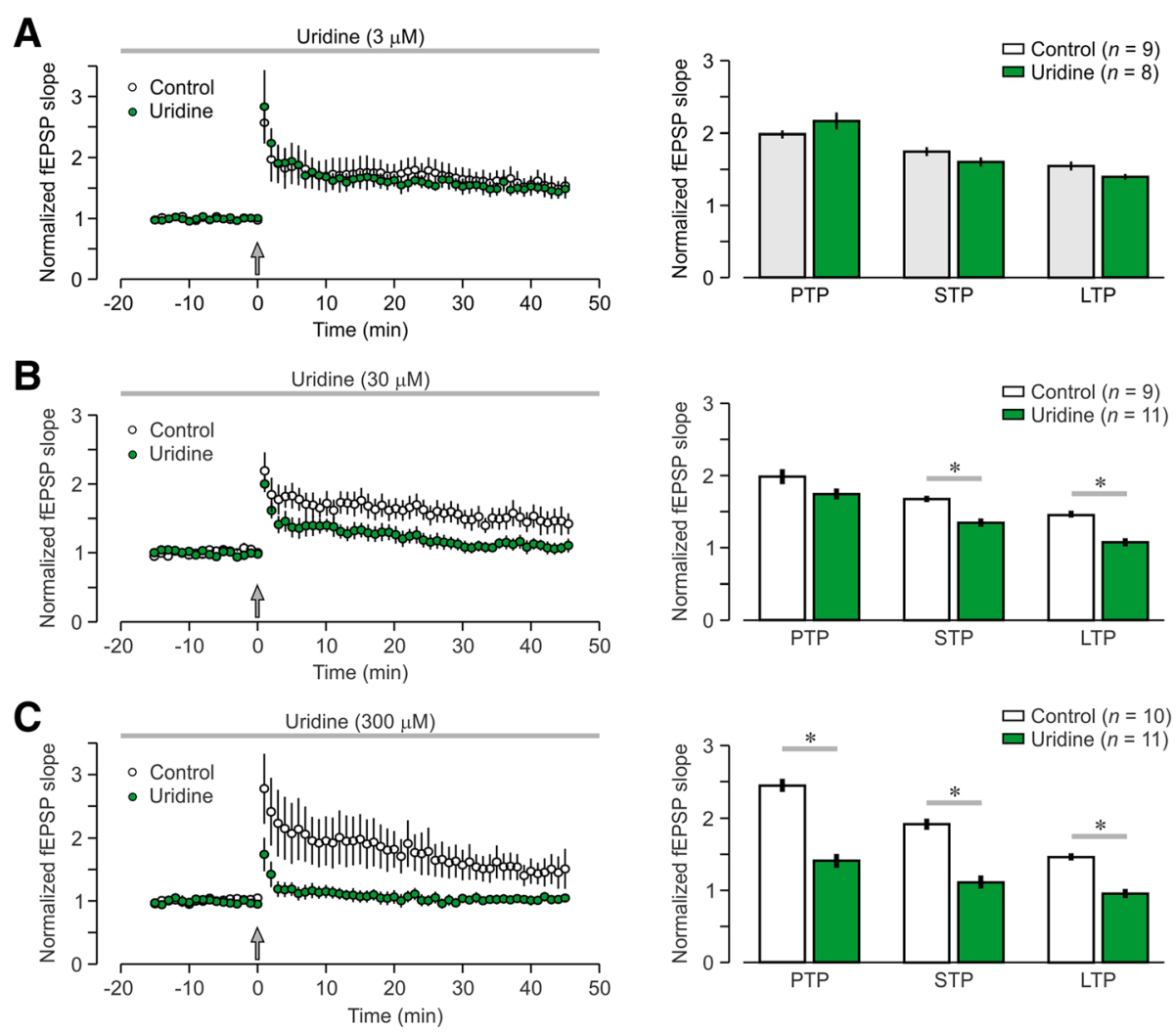

D

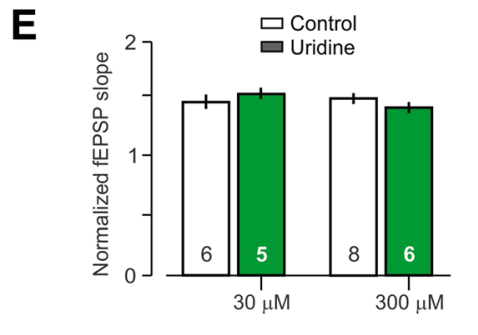

Fig. 3 Concentration-dependent effect of uridine on the induction of long-term potentiation. Brain slices are treated with uridine and fEPSPS are recorded for at least $15 \mathrm{~min}$ (baseline period). Then, HFS is delivered and fEPSPs are collected for an additional 45 min. Post-tetanic potentiation (PTP) is measured 1 min post-HFS, short-term potentiation (STP) is calculated 10-15 min post-HFS, and long-term potentiation (LTP) is measured 40-45 min post-HFS. a Left, graph showing the normalized fEPSP slopes (mean \pm SEM) for the uridine $(3 \mu \mathrm{M})$ and control groups; the arrow marks HFS. Right, bar graphs show that uridine $(3 \mu \mathrm{M})$ does not significantly affect any plasticity time point. $\mathbf{b}$ Uridine $(30 \mu \mathrm{M})$ has a lowering effect on STP and LTP, but PTP is unchanged; * $P<0.05$ (t-test). c Uridine $(300 \mu \mathrm{M})$ significantly decreases PTP, STP, and LTP; * $P<0.05$ (t-test). $\mathbf{d}$ Uridine $(300 \mu \mathrm{M})$ has a null effect on LTP expression, when introduced 10 min post-HFS, following the induction of LTP. e Graphs showing the negligible effect of uridine $(30 \mu \mathrm{M}, 300 \mu \mathrm{M})$ on LTP expression. Numbers within bars indicate number of brain slices per group

control, $110,878 \pm 10,838$; uridine, $117,452 \pm 11,146 \mathrm{~V}, U=$ $15, P=0.5$, MWU test) and was reduced, but not significantly, at the middle level of uridine $(30 \mu \mathrm{M}$; control, 118 , $617 \pm 14,944$; uridine, 88,924 $\pm 7398 \mathrm{~V}, U=33.5, P=0.079$, MWU test). Interestingly, the total depolarization at the high level of uridine $(300 \mu \mathrm{M})$ was significantly lower compared to controls (Fig. 4b; control, 114,507 $\pm 11,758$; uridine, $68,249 \pm 11,636 \mathrm{~V}, U=49, P<0.05$, MWU test). This suggests that the high level of uridine $(300 \mu \mathrm{M}) \mathrm{im}$ paired LTP induction, possibly by interacting with NMDARs during these high-frequency stimulation events.
NMDAR-mediated fEPSPs are reduced in amplitude by uridine

In order to measure a potential effect of uridine on NMDARs, we recorded pharmacologically isolated NMDAR-mediated fEPSPs in the absence and presence of uridine $(300 \mu \mathrm{M})$. Compared to typical fEPSPs, NMDAR-mediated fEPSPs were longer in duration, lower in amplitude, and were fully blocked by NMDAR antagonists (Faust et al., 2010; Izumi et al., 2006). Notably, we found that uridine $(300 \mu \mathrm{M})$ had an inhibitory effect on the amplitude of NMDAR-mediated fEPSPs (Fig. 4c, d). 


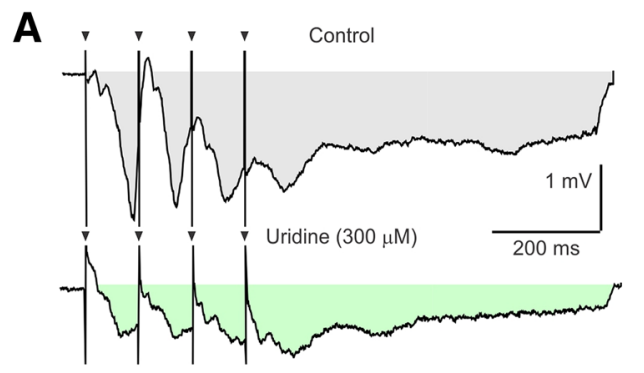

B

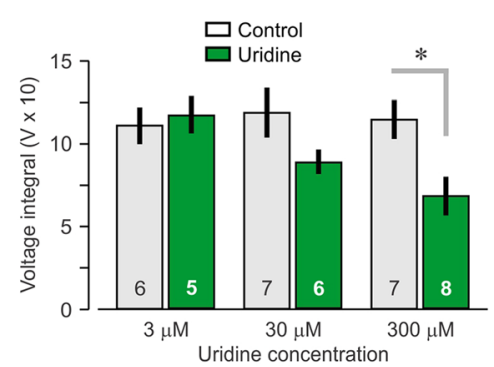

C

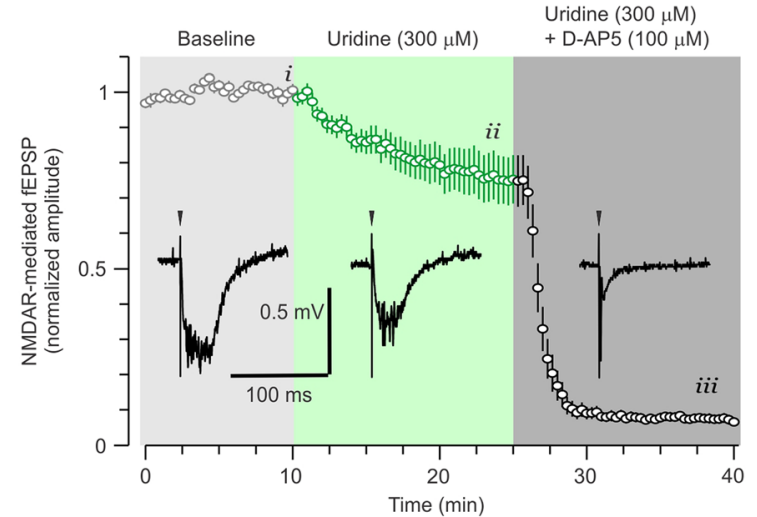

D

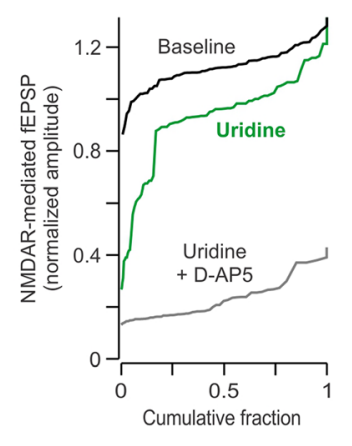

Fig. 4 Uridine decreases NMDAR-mediated synaptic responses. a Representative single theta-burst stimulation (TBS) event during LTP induction. Shaded areas indicate the total depolarization measured. b Mean total depolarization during TBS tetanus, which is highly mediated by NMDARs, is significantly lower in the presence of uridine $(300 \mu \mathrm{M}){ }^{*} P<0.05$ (MWU test). Numbers within bars indicate number of brain slices per group. $\mathbf{c}$ Plot shows mean fEPSP amplitudes of pharmacologically isolated NMDAR-mediated fEPSPs in several conditions: (i) baseline, no uridine (ii) uridine $(300 \mu \mathrm{M})$, and (iii) uridine $(300 \mu \mathrm{M})+$ D-AP5 $(100 \mu \mathrm{M})$. Accompanying sample traces from each condition are shown in the insets. $\mathbf{d}$ Cumulative probability plots show that uridine $(300 \mu \mathrm{M})$ significantly decrease NMDAR-mediated fEPSPs amplitudes, which are essentially eliminated in the presence of the NMDAR antagonist D-AP5

Mean NMDAR-mediated fEPSP amplitudes were lowered by $\sim 17 \%$ in the presence of uridine $(300 \mu \mathrm{M})$, compared to the baseline amplitudes (baseline, $0.176 \pm 0.012$; uridine, $0.146 \pm 0.013 \mathrm{mV}, D=0.7, P<0.0001$, KolmogorovSmirnov test). In order to verify that these fEPSPs were indeed NMDAR-mediated, we introduced the NMDARspecific antagonist D-2-amino-5-phosphonopentanoate (D-AP5), which eliminated the fEPSP almost entirely (mean amplitude in D-AP5 $=0.0135 \pm 0.003 \mathrm{mV}$ ). These results strongly suggest that uridine interacts with the NMDAR, acting as a partial antagonist or inhibiting agent. They also provide a mechanism to understand the LTP impairments we observed at the middle $(30 \mu \mathrm{M})$ and high $(300 \mu \mathrm{M})$ uridine levels.

\section{Protective effect of uridine against OGD}

To investigate the effect of uridine in an ex vivo model of brain insult, we used the OGD paradigm, which is known to trigger a rapid suppression of synaptic transmission. In this paradigm, synaptic responses fully recover (to $100 \%$ pre-insult) if the ischemic event is brief in duration. In order to obtain a reliable and reproducible OGD-induced deficit, we first ran a pilot study to test the effects of four different OGD durations; 4 min, $6 \mathrm{~min}, 8 \mathrm{~min}$, and $12 \mathrm{~min}$. As a result of this pilot work, we found that in our preparation a 6 min OGD challenge produced the most consistent fEPSP deficit with a mean maximum amplitude decrease of $46.0 \pm 5.6 \%$ that recovered back to baseline levels after $55.7 \pm 4.9 \mathrm{~min}$ (Fig. 5a). We then tested whether a relatively high dose of uridine $(100 \mu \mathrm{M})$ could alter this OGD-induced decrease in fEPSP amplitude. We tested three different uridine incubation periods: $15 \mathrm{~min}, 30 \mathrm{~min}$, and $45 \mathrm{~min}$. The incubation period was the amount of time uridine was present in the recording chamber before the OGD insult. We found that the $15 \mathrm{~min}$ uridine incubation did not result in a significantly different area-under-thecurve (AUC) measurement when compared to controls (data not shown). However, the 30-min uridine incubation period resulted in a significantly reduced the deficit (Fig. 5a), and the 45-min uridine incubation was even more effective (Fig. 5b, $T=5.39, P<0.001$, t-test). In order to appropriately quantify the OGD deficit and to compare the effect of uridine between groups, we measured the AUC of amplitude-by-time plots to generate a total OGD-deficit measure (Fig. 5c). Using this measure, 
A

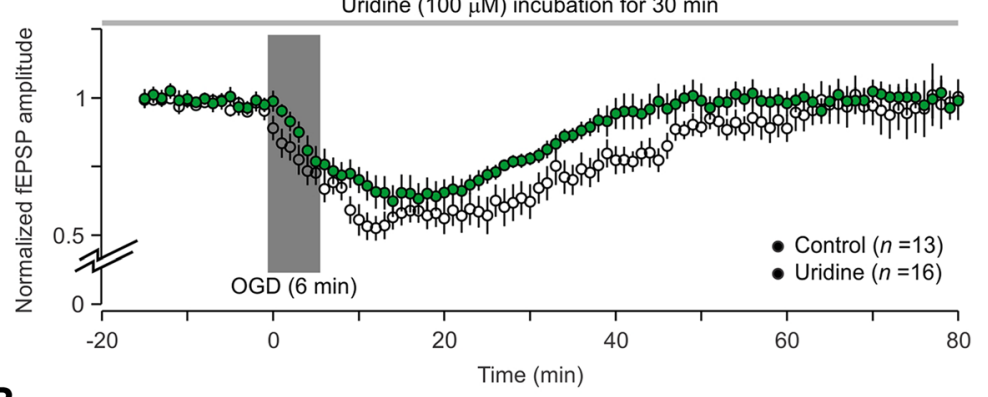

B
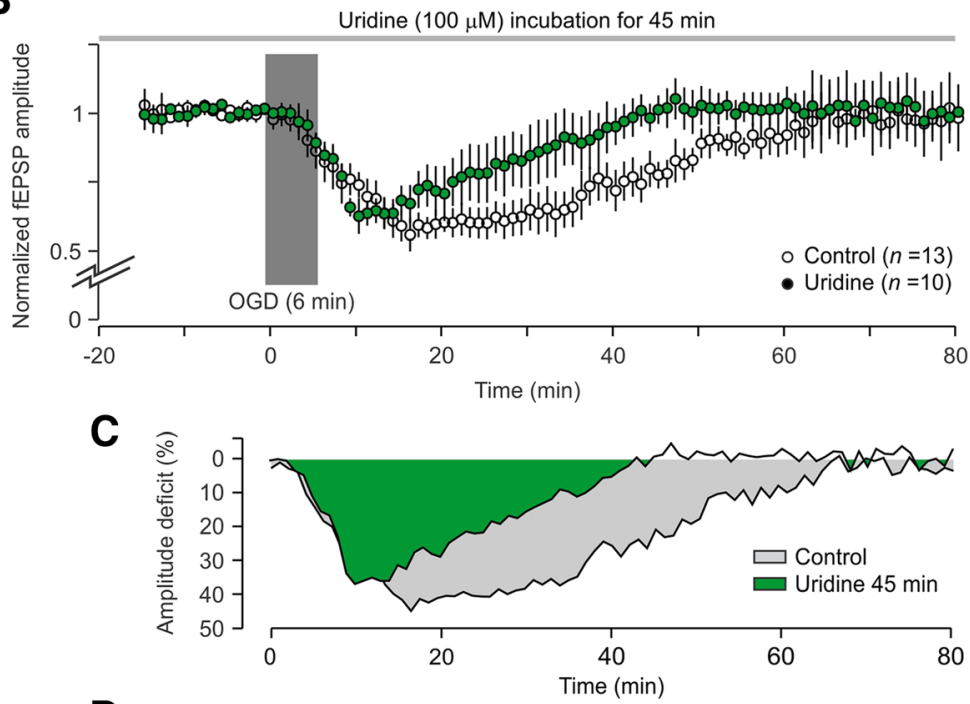

D

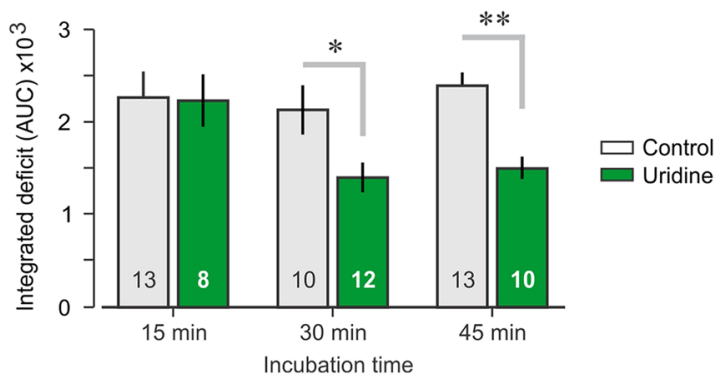

Fig. 5 Protective effect of uridine against oxygen-glucose deprivation. a Graph showing normalized fEPSP amplitudes (mean \pm SEM) for brain slices that are treated in uridine $(100 \mu \mathrm{M})$ for 30 min before receiving an oxygen-glucose deprivation $(\mathrm{OGD})$ insult $(6 \mathrm{~min})$. b In this set, brain slices are in uridine $(100 \mu \mathrm{M})$ for $45 \mathrm{~min}$ before receiving an OGD insult (6 min). c Plot showing the percent amplitude deficit for the 45 min uridine group compared to the untreated control group. In the uridine group, the amplitude deficit disappears by 45 min post-OGD (vs. 65 min in control), highlighting the protective action of uridine. $\mathbf{d}$ Graph showing the total OGD deficit, which is calculated from the percent amplitude deficit plots by taking the area-under-curve (AUC) for three different incubation periods: $15 \mathrm{~min}, 30 \mathrm{~min}$, and $45 \mathrm{~min}$. The short incubation (15 $\mathrm{min}$ ) is insufficient for a protective effect against 6 min OGD, while the longer incubation periods ( $30 \mathrm{~min}$ and $45 \mathrm{~min}$ ) significantly reduce the magnitude of the OGD-induced deficit; ${ }^{*}, P<0.05 ;{ }^{* *}, P<0.01$ (t-test). Numbers within bars indicate number of brain slices per group

we found that 15-min uridine incubation did not significantly affect the magnitude of the OGD deficit $(T=0.09$, $P=0.93$, t-test). For the longer incubation periods, we found that the deficit was significantly reduced by uridine incubation for $30 \mathrm{~min}$ (Fig. $5 \mathrm{~d}, T=2.77, P<0.01$, ttest) and $45 \mathrm{~min}$ (Fig. $5 \mathrm{~d}, T=5.39, P<0.001$, t-test), suggesting that uridine exerted a protective effect for the synaptic population against the OGD insult.

\section{Discussion}

Uridine has been investigated in a number of animal models for brain diseases (Amante et al., 2010; Cansev et al., 2008; De Bruin et al., 2003; Saydoff et al., 2006; Zhao et al., 2008), but despite this range of testing, the physiological effect of uridine on glutamatergic synaptic transmission and synaptic plasticity remains poorly understood. Using a set of electrophysiological assays in 
a brain slice preparation, our results demonstrate that uridine can impact glutamatergic synaptic transmission and synaptic plasticity in the mammalian brain. We have found that, at physiologically attainable concentrations within the brain $(30 \mu \mathrm{M}$ and $300 \mu \mathrm{M})$, uridine impairs long-term synaptic plasticity and inhibits NMDARmediated synaptic responses. Meanwhile, uridine does not have an effect on basal synaptic transmission or short-term synaptic plasticity. The protective action of uridine $(100 \mu \mathrm{M})$ against OGD insult indicates that it acts in a beneficial way to strengthen the synaptic population by diminishing the overall OGD-induced deficit.

Together these results are a step towards understanding the effect of uridine in the brain and may be important when evaluating molecular targets for neuromodulation or in the treatment of brain disorders. For example, these results may be relevant in disorders involving excessive glutamate levels such as hyperalgesia (Sandkühler, 2009), depression (Mitani et al., 2006), epilepsy (Meldrum, 1994), and stroke (Lai et al., 2014). Notably, the Food and Drug Administration (FDA) has already approved bioelectronic interventions, such as vagus nerve stimulation (VNS), for two of these disorders, intractable depression and intractable epilepsy. While the mechanism by which VNS reduces seizure frequency or ameliorates depressive symptoms is not understood, modulation of glutamate levels within the brain is one possibility (Ben-Menachem et al., 1995; Walker et al., 1999). Further highlighting the importance of glutamatergic modulation in treating certain brain disorders, the NMDAR antagonist ketamine was very recently approved by the FDA to treat intractable depression for its rapidly acting anti-depressive effects (Krystal et al., 2019; Serafini et al., 2014).

The inhibition of NMDAR-mediated fEPSPs and lower total depolarization during tetanus in the presence of uridine (Fig. 4) suggests that the LTP impairment (Fig. 3 ) is due to a reduction in NMDAR-induced calcium influx, subsequently leading to lower levels of synaptic potentiation (Morris et al., 1986; Tsien et al., 1996). A previous study reported that uridine inhibited calcium uptake into synaptosomes and acted as an inhibitor of pre-synaptic NMDARs (Petrova and Gabrelian, 2008). Our results corroborate this reduction in calcium influx and extend the effect to an inhibition of NMDARmediated synaptic responses. While the molecular mechanism by which uridine decreases NMDARmediated fEPSPs is not completely understood, the fact that the synaptic effects are not detectable until the isolation of NMDAR-specific potentials (Fig. 4) suggests that uridine may act as a noncompetitive antagonist, only interacting with NMDARs when they are being excessively activated. We found that uridine reduces total depolarization under NMDAR-only stimulation, but has no effect when AMPARs are primarily being activated, as is the case during basal synaptic transmission (Fig. 1). One possibility is that uridine does not compete directly for the glutamate-binding site on NMDARs, but functions as a noncompetitive antagonist to inhibit the NMDAR glycine-binding site (Johnson and Ascher, 1987). In fact, compounds that inhibit the glycinebinding site of NMDARs have previously shown neuroprotective effects in brain slice models of ischemia (Newell et al., 1995; Warner et al., 1995), similar to what we have reported here with uridine (Fig. 5). Since our LTP experiments were performed in the presence of the $\mathrm{GABA}_{\mathrm{A}}$ receptor inhibitor picrotoxin, we were not able to properly assess whether uridine interacted with the GABAergic system (Guarneri et al., 1985). However, we observed no empirical evidence that uridine displayed any GABA-mimetic effects, such as inhibiting basal synaptic transmission during our I-O tests (Fig. 1).

Excitotoxicity following a brain stroke is a primary mechanism of neuronal death and is associated with excessive glutamate that increases NMDAR-mediated calcium influx (Lai et al., 2014). While the molecular mechanisms of excitotoxicity remain poorly understood, excitatory glutamatergic transmission plays a central role in this pathophysiology. Electrophysiological measurements of glutamatergic brain activity, such as those used in this study, provide a reliable readout of neuronal and tissue viability that might be fundamental to the development of BEM treatments for stroke and other brain injuries (Rapp et al., 2015). Our observed protective effect of uridine against OGD-induced deficit (Fig. 5) may be attributed to the antagonism against NMDARs. As excitotoxic injury and activity are dependent on calcium influx via NMDARs, uridine may have attenuated this specific pathway for neuronal injury and thus allowed for a faster recovery following restoration of oxygen and glucose. It is also possible that the observed protective effect involved mechanisms that are independent of the decrease in NMDAR-mediated responses. These include possible bioenergetic effects and mitochondrial involvement (Geiger and Yamasaki, 1956). Since uridine is a pyrimidine nucleoside, the protective effects observed against OGD may be attributed to improving bioenergetics, such as elevating adenosine triphosphate (ATP) levels or enhancing glycolytic energy production. OGD triggers a rapid suppression of synaptic transmission that protects neurons by maintaining a minimal level of metabolism required for survival. This protective mechanism allows neurons to recover from ischemic insults of short duration, but prolonged ischemia ( $>10 \mathrm{~min}$ ) results in large increases in intracellular calcium, thus triggering cascades that lead irreversibly to cell death (Martin et al., 1994; Pugliese et al., 2003). Previous work has shown that uridine increases ATP levels following ischemic episodes in organs such as the heart (Aussedat, 
1983) and it has also been shown to prolong the normal homeostasis of brain tissue when added to perfusion fluids (Geiger, 1958). Therefore it is possible that uridine may be elevating ATP levels and signaling via purinergic receptors such as P2X receptors. P2X receptors are cation channels that are gated by ATP and can be found in various brain regions, including the hippocampus (North, 2002; Rubio and Soto, 2001; Skaper et al., 2009). These receptors are permeable to calcium and have been implicated in LTP processes with the potential to act as facilitators or inhibitors of plasticity, depending on the context (Pankratov et al., 2009; Wang et al., 2004). Future studies that include exploration of the ATP signaling system and the use of specific purinergic antagonists should be undertaken to elucidate the mechanism for this protective effect.

Our findings point to a potential benefit of uridine in the treatment of neurological disorders where glutamatergic systems are implicated and in cases where ischemia may be involved, such as stroke or traumatic brain injury (Rapp et al., 2015). However, there is growing evidence that glutamatergic systems also play a role in the pathophysiology of major depressive disorders (Sanacora et al., 2008; Sattler and Rothstein, 2007; Zarate et al., 2005). In fact, uridine has already shown efficacy in prior studies of depression (Carlezon et al., 2002, 2005) and clinical trials for bipolar disorder (Repligen 2006, 2008). Furthermore, preclinical studies with other pyrimidines that are similar to uridine have shown antidepressant properties with effectiveness either as monotherapy (Jensen et al., 2008) or in conjunction with other compounds such as valproate (Yoon et al., 2009). Indeed, there is evidence that patients suffering from mood disorders have increased levels of glutamate in certain brain regions (Hashimoto et al., 2007) and the NMDAR may be particularly important in susceptibility for these disorders (Mundo et al., 2008). The idea that mood disorders are a product of glutamatergic dysfunction is further bolstered by evidence that moodstabilizing drugs, such as valproate and lithium, exert neuroprotective effects against glutamate-induced excitotoxicity in neuronal cultures (Manji et al., 2000). Taken together, these pieces of evidence suggest that glutamatergic modulation of brain networks, whether by pharmacological means (e.g., uridine or ketamine) or by bioelectronic approaches (e.g., VNS), is efficacious for reducing symptoms of depression in a subset of patients.

Neuromodulation approaches using direct stimulation with implantable electrodes, such as deep brain stimulation (DBS), are a form of BEM that has been in clinical use for over two decades. DBS is effective for movement disorders, such as Parkinson's disease, and has also been investigated for the treatment of major depression (Williams and Okun, 2013). While DBS for these indications targets dopaminergic systems, similar neuromodulation technologies can be used to target glutamatergic systems. For instance, early clinical trials of DBS targeted to the fornix of Alzheimer's disease patients, were designed to increase glutamatergic activity in medial and corticolimbic brain circuits, with the explicit goal of improving cognition (Nardone et al., 2015). While larger scale clinical trials of fornix DBS did not show clinical efficacy in Alzheimer's disease (Leoutsakos et al., 2018), electrical neuromodulation of limbic structures such as the hippocampus remains an active area of investigation. Modulating hippocampal synaptic plasticity is often the goal of neurostimulation techniques, whether for the stabilization of memory decline in dementia or to ameliorate seizures in epilepsy. These emerging techniques require a thorough understanding of the excitatory brain networks and molecular targets that modulate them. Comprehensive electrophysiological testing of these circuits will improve our ability to intentionally alter them for therapeutic benefit.

As bioelectronic tools evolve and expand into CNS disorders involving glutamate, it will be important to understand the mechanisms of glutamatergic synaptic transmission and plasticity. It is also critically important to understand the role of potential neuromodulators, such as uridine, as targeted electronic interventions seek to replicate or improve upon traditional molecular targets. The electrophysiological assessment of glutamatergic systems, as demonstrated in this study, provides important foundational knowledge for the development of future BEM approaches aimed at treating a range of disorders involving glutamatergic signaling.

\section{Conclusions}

- Electrophysiological tests performed on brain slices can be used to identify specific alterations in glutamatergic synaptic transmission and plasticity

- Uridine is a nucleoside that affects NMDARmediated glutamatergic transmission.

- Uridine impairs short-term and long-term synaptic plasticity.

- OGD-induced synaptic transmission deficits are ameliorated by uridine.

- An improved understanding of glutamatergic brain systems, including mechanisms of neuromodulation, will be important for any bioelectronic approaches targeting these systems.

\section{Abbreviations}

ACSF: Artificial cerebral spinal fluid; AMPAR: a-amino-3-hydroxy-5-methyl-4isoxazolepropionic acid receptor; ATP: Adenosine triphosphate; AUC: Area under the curve; BALB/c: Bagg albino, genotype c; BEM: Bioelectronic medicine; CA1: Cornus Ammonis 1 area of the hippocampus; CA3: Cornus Ammonis 3 area of the hippocampus; CNQX: 6-cyano-7-nitroquinoxaline-2,3-dione; CNS: central nervous system; D-AP5: D-2-amino-5-phosphonopentanoate; 
DHA: docosahexaenoic acid; FDA: Food and Drug Administration; fEPSP: field excitatory postsynaptic potential; FV: fiber volley; GABA: gamma-aminobutyric acid; GLP: Good Laboratory Practice; HFS: high-frequency stimulation; I-O: inputoutput; IPI: inter-pulse interval; LTP: long-term potentiation; NMDAR: n-methyld-aspartate receptor; OGD: oxygen-glucose deprivation; P2X: purinergic ATPgated receptor 2X; PPF: paired-pulse facilitation; PTP: post-tetanic potentiation; RNA: ribonucleic acid; STP: short-term potentiation; TBS: theta-burst stimulation; VNS: vagus nerve stimulation

\section{Acknowledgements}

We are grateful to Steven W. Jones, James R. Rusche and Repligen Corporation for their invaluable help at the pilot stage of this study. We thank Seth Miller and Kelvin Chan for their excellent technical assistance.

\section{Authors' contributions}

EHC, STC, SAF, and PTH designed the experiments. EHC, SAF, and PTH performed experiments and analyzed the data. EHC and PTH made the final figures and wrote the manuscript. All authors approved the manuscript.

\section{Funding}

This work was supported by the National Institute of Health $(\mathrm{NIH})$ grant 5P01Al102852 and NIH grant 5P01Al073693 to PTH (project leader, Dr. Betty Diamond).

\section{Availability of data and materials}

The datasets used and analyzed during the current study are available from the corresponding author on reasonable request.

\section{Ethics approval}

All animal experimentation was performed in accordance with the National Institutes of Health $(\mathrm{NIH})$ Guidelines, under protocols approved by the Institutional Animal Care and Use Committee (IACUC) of the Feinstein Institute for Medical Research. Our Animal Research Program is registered with the Department of Health \& Human Services (DHHS), Office of Laboratory Animal Welfare (OLAW), United States Department of Agriculture (USDA \#21R0107), Public Health Service (PHS \#A3168-01) and New York State Department of Health (NYSDOH \#A-060).

\section{Consent for publication}

Not applicable.

\section{Competing interests}

The authors declare that they have no competing interests.

\section{Author details}

'Laboratory of Immune \& Neural Networks, Institutes of Molecular Medicine and Bioelectronic Medicine, Feinstein Institutes for Medical Research, Northwell Health, 350 Community Drive, Manhasset, NY 11030, USA.

${ }^{2}$ Laboratory of Biomedical Science, Institute of Bioelectronic Medicine, Feinstein Institutes for Medical Research, Northwell Health, 350 Community Drive, Manhasset, NY 11030, USA. ${ }^{3}$ Nimbus Therapeutics, 130 Prospect Street, Suite 301, Cambridge, MA 02139, USA. ${ }^{4}$ Department of Molecular Medicine, Zucker School of Medicine at Hofstra/Northwell, 500 Hofstra Blvd, Hempstead, NY 11549, USA.

\section{Received: 2 April 2019 Accepted: 20 May 2019}

Published online: 10 June 2019

\section{References}

Amante DJ, Kim J, Carreiro ST, Cooper A, Jones SW, Li T, Moody JP, Edgerly CK, Bordiuk OL, Cormier K, Smith K, Ferrante RJ, Rusche J. Uridine ameliorates the pathological phenotype in transgenic G93A-ALS mice. Amyotroph Lateral Scler. 2010:11:520-30.

Aussedat J. Effect of uridine supply on glycogen resynthesis after ischemia in the isolated perfused rat heart. Cardiovasc Res. 1983;17:145-51.

Ben-Menachem E, Hamberger A, Hedner T, Hammond EJ, Uthman BS, Slater J, Treig T, Stefan H, Ramsay RE, Wernicke JF, Wilder BJ. Effects of vagus nerve stimulation on amino acids and other metabolites in the CSF of patients with partial seizures. Epilepsy Res. 1995;20:221-7.

Bliss TV, Collingridge GL. A synaptic model of memory: long-term potentiation in the hippocampus. Nature. 1993;361:31-9.
Bliss TV, Lomo T. Long-lasting potentiation of synaptic transmission in the dentate area of the anaesthetized rabbit following stimulation of the perforant path. J Physiol. 1973;232:331-56.

Cansev M, Ulus IH, Wang L, Maher TJ, Wurtman RJ. Restorative effects of uridine plus docosahexaenoic acid in a rat model of Parkinson's disease. Neurosci Res. 2008:62:206-9.

Carlezon WA, Mague SD, Parow AM, Stoll AL, Cohen BM, Renshaw PF. Antidepressant-like effects of uridine and omega-3 fatty acids are potentiated by combined treatment in rats. Biol Psychiatry. 2005;57:343-50.

Carlezon WA, Pliakas AM, Parow AM, Detke MJ, Cohen BM, Renshaw PF. Antidepressant-like effects of cytidine in the forced swim test in rats. Biol Psychiatry. 2002;51:882-9.

Cho S, Wood A, Bowlby MR. Brain slices as models for neurodegenerative disease and screening platforms to identify novel therapeutics. Curr Neuropharmacol. 2007:5:19-33.

Dawson DM. Enzymatic conversion of uridine nucleotide to cytidine nucleotide by rat brain. J Neurochem. 1968;15:31-4.

De Bruin NM, Kiliaan AJ, De Wilde MC, Broersen LM. Combined uridine and choline administration improves cognitive deficits in spontaneously hypertensive rats. Neurobiol Learn Mem. 2003;80:63-79.

Faust TW, Chang EH, Kowal C, Berlin R, Gazaryan IG, Bertini E, Zhang J, SanchezGuerrero J, Fragoso-Loyo HE, Volpe BT, Diamond B, Huerta PT. Neurotoxic lupus autoantibodies alter brain function through two distinct mechanisms. Proc Natl Acad Sci U S A. 2010;107:18569-74.

Geiger A. Correlation of brain metabolism and function by the use of a brain perfusion method in situ. Physiol Rev. 1958;38:1-20.

Geiger A, Yamasaki S. Cytidine and uridine requirement of the brain. J Neurochem. 1956;: :93-100.

Guarneri P, Guarneri R, La Bella V, Piccoli F. Interaction between uridine and GABA-mediated inhibitory transmission: studies in vivo and in vitro. Epilepsia. 1985;26:666-71.

Hashimoto K, Sawa A, lyo M. Increased levels of glutamate in brains from patients with mood disorders. Biol Psychiatry. 2007:62:1310-6.

Izumi Y, Auberson YP, Zorumski CF. Zinc modulates bidirectional hippocampal plasticity by effects on NMDA receptors. J Neurosci. 2006;26:7181-8.

Jensen JE, Daniels M, Haws C, Bolo NR, Lyoo IK, Yoon SJ, Cohen BM, Stoll AL, Rusche JR, Renshaw PF. Triacetyluridine (TAU) decreases depressive symptoms and increases brain $\mathrm{pH}$ in bipolar patients. Exp Clin Psychopharmacol. 2008;16:199-206.

Johnson AW, Ascher P. Glycine potentiates the NMDA response in cultured mouse brain neurons. Nature. 1987;325:529-31.

Kabadi SV, Maher TJ. Post treatment with uridine and melatonin following traumatic brain injury reduces edema in various brain regions in rats. Ann $\mathrm{N}$ Y Acad Sci. 2010;1199:105-13.

Krystal JH, Abdallah CG, Sanacora G, Charney DS, Duman RS. Ketamine: a paradigm shift for depression research and treatment. Neuron. 2019;101: $774-8$.

Lai TW, Zhang S, Wang YT. Excitotoxicity and stroke: identifying novel targets for neuroprotection. Prog Neurobiol. 2014;115:157-88.

Leoutsakos JS, Yan H, Anderson WS, Asaad WF, Baltuch G, Burke A, Chakravarty MM, Drake KE, Foote KD, Fosdick L, Giacobbe P, Mari Z, McAndrews MP, Munro CA, Oh ES, Okun MS, Pendergrass JC, Ponce FA, Rosenberg PB, Sabbagh MN, Salloway S, Tang-Wai DF, Targum SD, Wolk D, Lozano AM, Smith GS, Lyketsos CG. Deep brain stimulation targeting the fornix for mild Alzheimer dementia (the ADvance trial): a two year follow-up including results of delayed activation. J Alzheimers Dis. 2018;64:597-606.

Manji HK, Moore GJ, Rajkowska G, Chen G. Neuroplasticity and cellular resilience in mood disorders. Mol Psychiatry. 2000;5:578-93.

Martin RL, Lloyd HG, Cowan Al. The early events of oxygen and glucose deprivation: setting the scene for neuronal death? Trends Neurosci. 1994;17: 251-7.

Meldrum BS. The role of glutamate in epilepsy and other CNS disorders. Neurology. 1994;44(11 Suppl 8):S14-23.

Mitani H, Shirayama Y, Yamada T, Maeda K, Ashby CR, Kawahara R. Correlation between plasma levels of glutamate, alanine and serine with severity of depression. Prog Neuro-Psychopharmacol Biol Psychiatry. 2006;30:1155-8.

Morris RGM, Anderson E, Lynch GS, Baudry M. Selective impairment of learning and blockade of long-term potentiation by an N-methyl-D-aspartate receptor antagonist. Nature. 1986;319:774-6.

Mundo E, Tharmalingham S, Neves-Pereira M, Dalton EJ, Macciardi F, Parikh SV, Bolonna A, Kerwin RW, Arranz MJ, Makoff AJ, Kennedy JL. Evidence that the 
N-methyl-D-aspartate subunit 1 receptor gene (GRIN1) confers susceptibility to bipolar disorder. Mol Psychiatry. 2008;8:241-5.

Nardone R, Höller Y, Tezzon F, Christova M, Schwenker K, Golaszewski S, Trinka E, Brigo F. Neurostimulation in Alzheimer's disease: from basic research to clinical applications. Neurol Sci. 2015;36:689-700.

Newell DW, Barth A, Malouf AT. Glycine site NMDA receptor antagonists provide protection against ischemia-induced neuronal damage in hippocampal slice cultures. Brain Res. 1995;675:38-44.

North RA. Molecular physiology of P2X receptors. Physiol Rev. 2002;82:1013-67.

Pankratov Y, Lalo U, Krishtal OA, Verkhratsky A. P2X receptors and synaptic plasticity. Neuroscience. 2009;158:137-48.

Petrova LN, Gabrelian AV. Effect of uridine of presynaptic NMDA and kainate receptor of rat brain cortex. Bull Exp Med. 2008;145:320-2.

Pooler AM, Guez DH, Benedictus R, Wurtman RJ. Uridine enhances neurite outgrowth in nerve growth factor-differentiated PC 12 (corrected). Neuroscience. 2005;134:207-14.

Pugliese AM, Latini S, Corradetti R, Pedata F. Brief, repeated, oxygen-glucose deprivation episodes protect neurotransmission from a longer ischemic episode in the in vitro hippocampus: role of adenosine receptors. $\mathrm{Br} \mathrm{J}$ Pharmacol. 2003;140:305-14

Rapp PE, Keyser DO, Albano A, Hernandez R, Gibson DB, Zambon RA, Hairston WD, Hughes JD, Krystal A, Nichols AS. Traumatic brain injury detection using electrophysiological methods. Front Hum Neurosci. 2015;9:11. https://doi.org/ 10.3389/fnhum.2015.00011.

Repligen. Phase II study to assess RG2417 in the treatment of bipolar I depression. 2006. In: ClinicalTrials.gov [Internet]. Bethesda, MD: National Library of Medicine (US). Available from: http://clinicaltrials.gov/show/ NCT00322764. NLM Identifier: NCT00322764. Accessed 28 May 2019.

Repligen. A study to assess the safety, tolerability and efficacy of RG2717 in bipolar I depression. 2008. In: ClinicalTrials.gov [Internet]. Bethesda, MD: National Library of Medicine (US). Available from: http://clinicaltrials.gov/ show/NCT00812058. NLM Identifier: NCT00812058. Accessed 28 May 2019.

Rubio ME, Soto F. Distinct localization of P2X receptors at excitatory postsynaptic specializations. J Neurosci. 2001;21:641-53.

Sanacora G, Zarate CA, Krystal JH, Manji HK. Targeting the glutamatergic system to develop novel, improved therapeutics for mood disorders. Nat Rev Drug Discov. 2008;7:426-37.

Sandkühler J. Models and mechanisms of hyperalgesia and allodynia. Physiol Rev. 2009;89:707-58

Sattler R, Rothstein JD. Targeting an old mechanism in a new disease-protection of glutamatergic dysfunction in depression. Biol Psychiatry. 2007;61:137-8.

Saydoff JA, Garcia RA, Browne SE, Liu L, Sheng J, Brenneman D, Hu Z, Cardin S, Gonzalez A, von Borstel RW, Gregorio J, Burr H, Beal MF. Oral uridine prodrug PN401 is neuroprotective in the R6/2 and N171-82Q mouse models of Huntington's disease. Neurobiol. Dis. 2006;24:455-65.

Serafini G, Howland RH, Rovedi F, Girardi P, Amore M. The role of ketamine in treatment-resistant depression: a systematic review. Curr Neuropharmacol. 2014;12:444-61.

Silei V, Politi V, Lauro GM. Uridine induces differentiation in human neuroblastoma cells via protein kinase C epsilon. J Neurosci Res. 2000;61: 206-11.

Skaper SD, Debetto P, Giusti P. The P2X7 purinergic receptor: from physiology to neurological disorders. FASEB. 2009;24:337-45.

Slezia A, Kekesi AK, Szikra T, Papp AM, Nagy K, Szente M, Magloczky Z, Freund TF, Juhasz $G$. Uridine release during aminopyrimidine-induced epilepsy. Neurobiol Dis. 2004;16:490-9.

Tsien J, Huerta PT, Tonegawa S. The essential role of hippocampal CA1 NMDA receptor-dependent synaptic plasticity in spatial memory. Cell. 1996;87:1327-38.

van Groeningen CJ, Peters GJ, Nadal JC, Laurensse E, Pinedo HM. Clinical and pharmacologic study of orally administered uridine. J Natl Cancer Inst. 1991; 83:437-41.

Walker BR, Easton A, Gale K. Regulation of limbic motor seizures by GABA and glutamate transmission in nucleus tractus solitarius. Epilepsia. 1999;40:1051-7.

Wang L, Albrecht MA, Wurtman RJ. Dietary supplementation with uridine-5'monophosphate (UMP), a membrane phosphatide precursor, increases acetylcholine level and release in striatum of aged rat. Brain Res. 2007;1133: $42-8$.

Wang L, Pooler AM, Albrecht MA, Wurtman RJ. Dietary uridine-5'monophosphate supplementation increases potassium-evoked dopamine release and promotes neurite outgrowth in aged rats. J Mol Neurosci. 2005; 27:137-45.
Wang Y, Haughey NJ, Mattson MP, Furukawa K. Dual effects of ATP on rat hippocampal synaptic plasticity. Neuroreport. 2004;15:633-6.

Warner DS, Martin H, Ludwig P, McAllister A, Keana JF, Weber E. In vivo models of cerebral ischemia: effects of parentally administered NMDA receptor glycine site antagonists. J Cereb Blood Flow Metab. 1995;15:188-96.

Weinberg ME, Roman MC, Jacob P, Wen M, Cheung P, Walker UA, Mulligan K, Schambelan M. Enhanced uridine bioavailability following administration of a triacetyluridine-rich nutritional supplement. PLoS One. 2010;6:e14709. https:// doi.org/10.1371/journal.pone.0014709.

Williams NR, Okun MS. Deep brain stimulation in the treatment of depression. J Clin Invest. 2013;123:4546-56.

Wurtman RJ, Cansev M, Sakamoto T, Ulus I. Nutritional modifiers of aging brain function: use of uridine and other phosphatide precursors to increase formation of brain synapses. Nutr Rev. 2010;68(Suppl 2):S88-101.

Yoon SJ, Lyoo IK, Haws C, Kim TS, Cohen BM, Renshaw PF. Decreased glutamate/ glutamine levels may mediate cytidine's efficacy in treating bipolar depression: a longitudinal proton magnetic resonance spectroscopy study. Neuropsychopharmacology. 2009;34:1810-8.

Zarate CA, Singh J, Manji HK. Cellular plasticity cascades: targets for the development of novel therapeutics for bipolar disorder. Biol Psychiatry. 2005: 59:1006-20.

Zhao Q, Marolewski A, Rusche JR, Holmes GL. Effects of uridine in models of epileptogenesis and seizures. Epilepsy Res. 2006;70:73-82.

Zhao Q, Shatskikh T, Marolewski A, Rusche JR, Holmes GL. Effects of uridine on kindling. Epilepsy Behav. 2008;13:47-51.

Zucker RS. Short-term synaptic plasticity. Annu Rev Neurosci. 1989;12:13-31.

\section{Publisher's Note}

Springer Nature remains neutral with regard to jurisdictional claims in published maps and institutional affiliations.

Ready to submit your research? Choose BMC and benefit from:

- fast, convenient online submission

- thorough peer review by experienced researchers in your field

- rapid publication on acceptance

- support for research data, including large and complex data types

- gold Open Access which fosters wider collaboration and increased citations

- maximum visibility for your research: over $100 \mathrm{M}$ website views per year

At $\mathrm{BMC}$, research is always in progress.

Learn more biomedcentral.com/submissions 